\title{
Efficiency multipliers for construction productivity: A Comparative Evaluation
}

DOI 10.5592/otmcj.2015.1.3

Research paper

\author{
Antonios Panas \\ Centre for Construction Innovation, \\ Department of Construction Engineering \\ and Management, Faculty of Civil \\ Engineering, National Technical University \\ of Athens, Athens, Greece \\ cvapanas@mail.ntua.gr
}

\author{
John-Paris Pantouvakis \\ Centre for Construction Innovation, \\ Department of Construction Engineering \\ and Management, Faculty of Civil \\ Engineering, National Technical University \\ of Athens, Athens, Greece \\ jpp@central.ntua.gr
}

\section{Keywords}

Concrete pavements, Estimation, Excavation, Multipliers, Productivity.
EFFICIENCY MULTIPLIERS FOR CONSTRUCTION PRODUCTIVITY ARE OFTEN ESTIMATED ON AN AD-HOC BASIS, DEPENDING ON THE PROJECT CHARACTERISTICS. The purpose of the study is to define a structured approach allowing the determination of the appropriate empirical productivity relations and efficiency multipliers along with their respective values. The proposed method breaks down a given construction activity into distinct operational scenarios which represent unique combinations of key productivity variables, thus providing a perspective on construction productivity for both labor-intensive and equipment-intensive operations. In addition, this is the first study to explicitly describe the process and the theoretical prerequisites for the statistically valid derivation and comparative evaluation of new efficiency multipliers for a given construction activity. A case study of heavy-duty concrete paving activities over an eight month period is utilized as a testbed for the derivation of new efficiency multipliers. An excavation scenario with the use of published estimation formulae is also presented to demonstrate the approach's capability to corroborate the values of known efficiency multipliers. The results indicate that the proposed approach improves the accuracy of estimated multipliers stemming from past productivity studies and increases the estimation precision for the derivation of new multipliers related to future construction operations. 


\section{INTRODUCTION}

Construction productivity is one of the main drivers for completing projects within time and cost limitations (Moselhi and Khan, 2010) and as such its appropriate estimation is quite important for preparing construction schedules and budgets (Song and AbouRizk, 2008). To determine construction productivity one needs to estimate an average production rate (Kiziltas and Akinci, 2009; Song and AbouRizk 2008) and then adjust it to the specific operational conditions of the job, such as temperature, overall site organization, crew skill, on the job learning for repetitive work (Panas and Pantouvakis, 2014) etc by multiplying it by a set of "efficiency coefficients" or "efficieny multipliers" (AbouRizk et al., 2001).

The average productivity is estimated using published formulae proposed either by manufacturers such as Caterpillar (Caterpillar, 2014) and Komatsu (Komatsu, 2009) or by widely acknowledged and accepted institutions such as the BML (1983). It should be noted that in some cases and for certain construction operations there are no published formulae in the literature (Panas and Pantouvakis, 2010). In these situations, one should determine the procedural framework allowing the incorporation of an initially unknown but defined later, during the process, set of operational factors (Pantouvakis and Panas, 2013).

The average productivity is then multiplied by appropriate "efficiency multipliers" whose determination, however, is not trivial as the relationship between the affecting factors and productivity is not well understood (O'Connor and Huh 2006). Different methods may suggest different sets of efficiency multipliers for the same operation each of which may take values from a specific range of expected values. The selection of values suggested by manufacturers is somewhat vague, as explicit guidelines for the selection of these values are not available (Jang et al., 2011; Moselhi and Khan, 2012) and, furthermore, may lead to unrealistically optimistic results (Lambropoulos et al., 1996). Also, the estimators cannot verify the accuracy of the efficient multipliers selected for the particular operations under study.

The above shortcomings are addressed in this paper. More specifically, the research objectives may now be stated as follows:

(i) To define a structured approach allowing the determination of the appropriate empirical productivity relations and efficiency multipliers along with their respective values.

(ii) To validate the above approach on selected operations of a real-world large-scale infrastructure project for both labor-intensive and equipmentintensive operations.

The structure of the paper is as follows: The following section presents background information on construction productivity. Then we proceed with reviewing basic information on the concrete paving process, which will be later used as a testbed for the approach proposed herein, from a labor-intensive operations perspective. In addition, the productivity estimation formulae for excavation operations with the use of hydraulic excavator is presented, as an exemplar of equipment-intensive operations. The research methodology is discussed in the subsequent section. The next section exemplifies the approach for the estimation of new multipliers by analyzing heavy-duty concrete pavement construction operations. The analysis' results stemming from field measurements are reported along with the main factors and efficiency multipliers that affect the achieved productivity. Then, the approach is implemented for the corroboration of known efficiency multipliers, by examining an excavation scenario. The main inferences emerging from the study are discussed and, finally, the conclusions and future directions for research are delineated.

\section{Background}

\section{Literature review}

In spite of the extensive research on construction productivity, there is no standard definition for its estimation (Moselhi and Khan, 2012). Therefore, this research defines construction productivity as the ratio of work-hours per output (e.g. wh/m³), which is often called the "unit rate" (Thomas and Yiakoumis, 1987). The scope of the analysis is set at the crew level, so as to examine factors that pose short-term variations on productivity on a daily basis (Moselhi and Khan, 2010). A measure of productivity which has been long used in the estimating process is the performance ratio (PR), whose mathematical expression is given as follows (Thomas and Yiakoumis, 1987) (see Equation 1):

$\mathrm{PR}=$ Effective Productivity / Theoretical Productivity

Usually, the effective or actual productivity is worse than the theoretical estimate, so in most cases the PR is lower than 1.00. In the estimating process, the expected productivity rates are generally tabulated as average values reflecting average conditions for a given project (AbouRizk et al., 2001). Thus, single-value estimates of productivity are typically used in preparing a bid. Therefore, if the PR is known in advance, then the estimation's accuracy will increase. The performance ratio may be regarded as an efficiency multiplier, since it is an aggregate measure that incorporates the effect of several factors (AbouRizk et al., 2001; Thomas and Yiakoumis, 1987).

However, a review of pertinent research reveals some key limitations in the estimation of the efficiency multipliers. First, the number of factors affecting productivity and the magnitude of their impact within a project varies (Hasan et al., 2013). Hence, there is a difficulty in properly considering all factors that impact productivity for a given activity (AbouRizk et al., 
2001). Therefore, the efficiency multipliers must be directly associated with a specific productivity factor, so as to explicitly determine both the scope of the analysis, as well as the limitations in generalizing the applicability of the estimated outputs. Secondly, the use of an aggregate measure of PR quantifies the combined impact of all considered factors on the production rate, but limits the ability to isolate the impact of any single factor from others (O'Connor and Huh, 2006).

Consequently, it would be useful for the PR to be further analyzed in its constituent factors, in order to gain a more detailed insight on the drivers that shape the effective productivity.

Thirdly, a proper projection of the condition that each factor will assume when the job commences and the extent of their impact on productivity have still not been adequately addressed in literature (AbouRizk et al., 2001). In that sense, the correct determination of each factor's state in a multi-factor productivity analysis is of major significance.

In view of the aforementioned, it is clear that the PR is a dynamically changing measure of productivity that depends on the type and size of the productivity factors involved in the estimating process. As such, in this paper we suggest a modification to Equation 1 to provide for the multifaceted effect of the varying productivity factors as

i $n^{Q_{\text {eff }}}=Q_{\text {th }} \times P R=Q_{\text {th }} \times \prod_{i=1}^{f} p_{i}$ show Equation 2 below.

where: $\mathrm{Q}_{\text {eff/th }}=$ effective/theoretical productivity for a given activity; $\mathrm{PR}=$ performance ratio; $\mathrm{p}_{\mathrm{i}}=$ efficiency multiplier corresponding to productivity factor $i$ for the adjustment of theoretical to effective productivity; and $\mathrm{f}=$ number of productivity factors.

In essence, as shown in Equation 2, PR decomposes into a set of multipliers each of which represents the effect of a specific productivity factor (e.g. weather) on productivity. Factors not affecting productivity assume a value equal to 1.00 . Similarly, if all factors are considered equal to 1.00 , then the theoretical and effective productivities coincide.

How do we determine the set of multipliers required and their respective values in each case? Clearly, we need a methodology, which we will present and discuss in some detail in section 3 of this paper. We also need at least two construction operations to exemplify the approach; one with a known and one with unknown average productivity formulae. We review briefly these construction operations in the following paragraphs.

\section{Selection of construction operations}

The approach proposed in this study to estimate and compare the $p_{i}$ coefficients for a given construction activity will be exemplified through the test application in two construction activities; a labour intensive one where the average productivity formulae is not known and an equipment intensive one with a known average productivity formulae. For the former, we have selected the complex concrete paving operation, whereas for the latter we have opted for the common excavation operation using a hydraulic excavator.

\section{Concrete paving operations}

Concrete paving operations require the combination of both equipment- and labor-intensive resources, with a particular focus on the latter. Published productivity data are scarce and based mainly on road construction. For the purposes of this paper, we consider the concrete pavement construction process to encompass area marking and preparation, concrete pouring, concrete layering, concrete finishing and joints cutting operation. More specifically, the layering of ready-mixed concrete for the construction of heavy-duty surfaces in external areas, such as those required for the loading operations in harbours performed by large cranes, will be examined herein (Figure 1). See Panas and Pantouvakis (2011) for further information on the construction process.

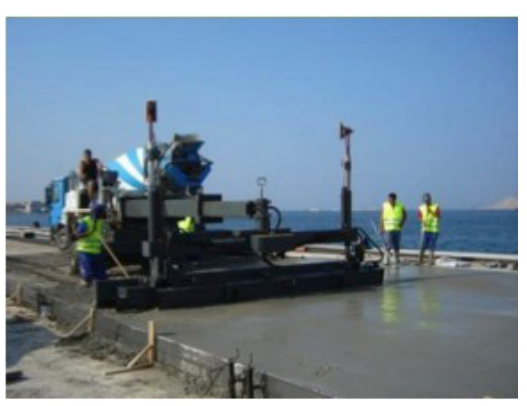

Figure 1. Layering of ready-mixed concrete for the construction of heavy duty surfaces.

\section{Common excavating operations}

One of the most well-known construction operations is excavation using a hydraulic excavator. For this operation, there are many published methods for productivity estimation. Here we adopt one of the most widely accepted by construction practitioners, namely the one defined in BML (1983). Based on Panas and Pantouvakis (2010), we may calculate $Q_{\text {eff }}$ for this operation by Equations $3 a, b$ and $c$ : $Q_{\text {eff }}=Q_{\text {th }} \times p_{\text {swing }} \times p_{\text {depth }}$ (3a) $\mathrm{p}_{\text {swing }}=4 \times 10^{-6} \times \mathrm{s}_{\mathrm{a}}^{2}-0.0024 \times \mathrm{s}_{\mathrm{a}}$ $+1.1824$

$p_{\text {depth }}=0.0043 \times d^{2}-0.0622 \times d+$ 1.0618

where: $d=$ excavation depth [m]; $\mathrm{p}_{\text {swing/depth }}=$ the dedicated efficiency multiplier representing the quantitative impact of the swing angle and excavation depth for the adjustment of theoretical to effective productivity; and $\mathrm{s}_{\mathrm{a}}=$ swing angle $\left[{ }^{\circ}\right]$.

Equipped with the basic theoretical background and a selection of appropriate construction operations, we can now present the research methodology and demonstrate its application on the selected processes. 


\section{Research methodology}

The research methodology comprises of three main phases; data elicitation, productivity model generation and efficiency multipliers determination, as summarized in Figure 2 and presented in the following paragraphs.

\section{Data elicitation}

The first step of the data elicitation process is the definition of the activity that is going to be studied. Flowcharts are drawn, so as to decompose each activity in its " $n$ " constituent sub-tasks $\left(s_{i}\right)$ and reveal the interactions between them. The scope of the experimental framework should be defined for every sub-task, including contextual information, such as location of the site, project characteristics, deployed resources etc. Following the definition of the context within which the study will be conducted, the operational factors affecting productivity should be specified. As mentioned in the previous section, each one of the identified sub-tasks can be completed at a certain theoretical productivity level. However, it is evident that each project is different and, thus, deviations from theoretical values are expected, leading to the actually effective productivity achieved on site. In that sense, the term "operational" denotes specific micro-level factors that can directly influence the effective productivity of any construction operation. The factors are conceptualised by measuring specific physical parameters (i.e. excavation depth, concrete pouring volume etc.) or by using a categorical variable, in case of qualitative factors (e.g. crew skill). Their influence is quantified by the use of the respective productivity efficiency multipliers $\left(p_{i}\right)$, whose mathematical formalisation is provided by Equation 2. As such, the study will be focused on scrutinising the impact of key factors to productivity by measuring parameters that are believed to shape the values of the productivity efficiency multipliers $\left(p_{i}\right)$.
The next step is the elicitation of work study data on a daily basis through the utilization of direct observation techniques, enhanced by the study of ancillary data, such as contractual documents, project reports, workhours logs, interviews with key project staff etc. One daily measurement corresponds to one data point (DP) and the $\mathrm{m}$ elements or collected DP's for a specific sub-task constitutes one dataset (D) (see Equation 4):

$\forall s_{i} \Rightarrow D_{i}=\left\{D P_{1}, D P_{2} \ldots, D P_{m}\right\}, i \leq n, i, n, m \in N$

Regarding the sample size, as the number of data points in each dataset increases, the validity of the analysis is potentially improved.

\section{Productivity model generation}

Productivity models can be generated by adopting data-oriented techniques (e.g. statistical regression, artificial neural networks), where the collected data are directly associated with each

1. Data elicitation

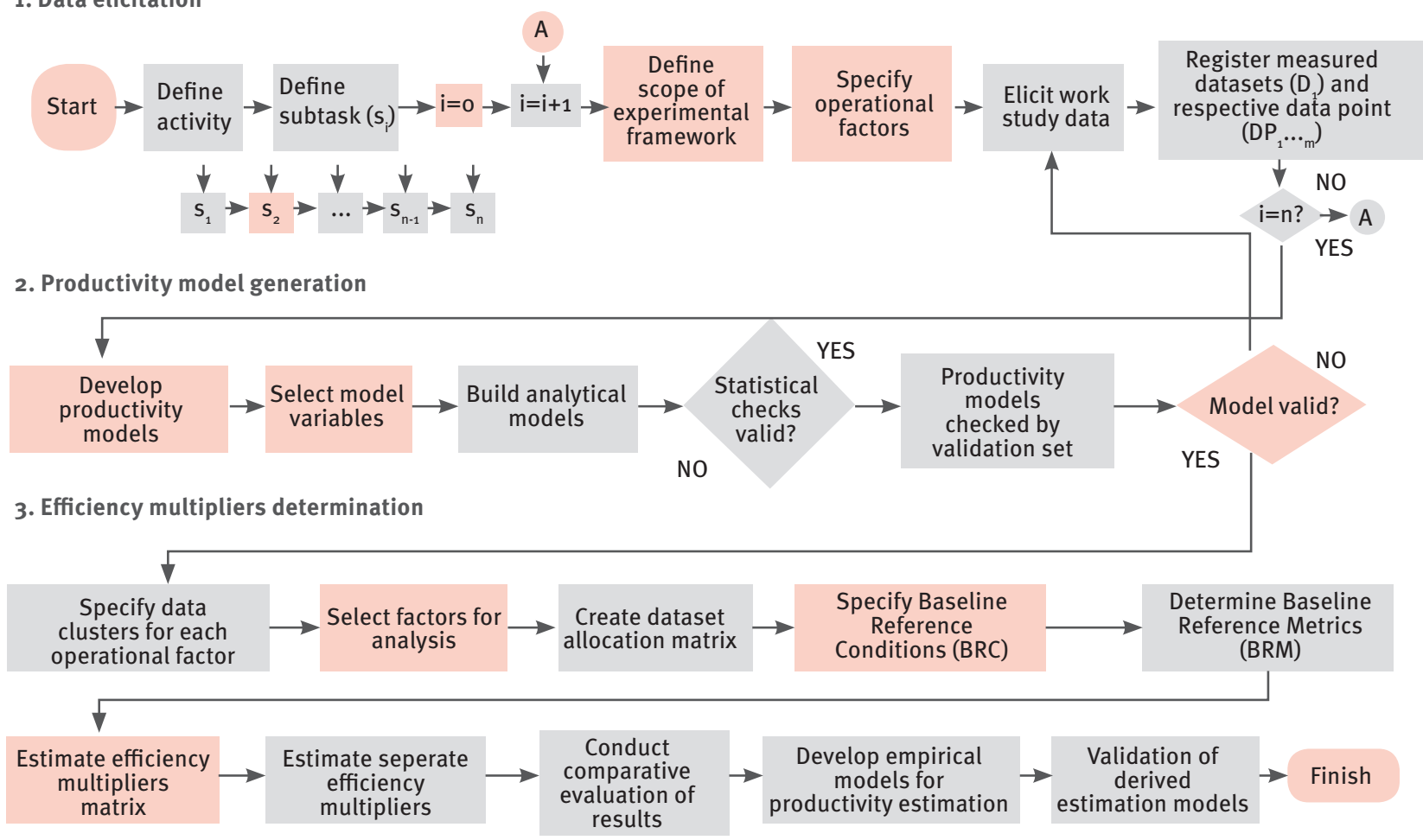

Figure 2: Research methodology. 
other, without considering the process behind this data. Irrespective of the selected technique, the model's variables should be determined in the following manner: productivity should be regarded as the response variable, whereas the individual productivity factors (i.e. efficiency multipliers) are the model's independent variables. Upon the performance of the required statistical checks that ensure the model's robustness, the validation process initiates. The validation process is performed by comparing the outputs of the developed models to the actual collected data. Hence, the validation process includes the substitution of validation data inputs to the designed models, so as to compare predicted results of the productivity models to the collected data. The statistical regression approach is adopted in this study and the reader is referred to Panas and Pantouvakis (2011) for more details.

Efficiency multipliers determination This section serves the main objectives of the study, in terms of the research contribution. The key issue is the establishment of a valid experimental framework which will consequently help the categorization and the in-depth analysis of the data within the regression models. First, every operational factor is divided in specific categories, or clusters, whose range is decided by the analyst. The limits of all clusters are denoted by the minimum and maximum values or the ordinal values for every quantitative (e.g. min and max value of working length) and qualitative factor (e.g. fiber-reinforced or plain concrete) respectively. A sub-set of the measured factors is chosen in pairs for further elaboration. A dataset allocation table is created, including all datapoints of a given sub-task's dataset, as follows (see Table 1).

For validity reasons, all subsets contained in each table cell should sum up to the initial dataset (see Equation 5):

\begin{tabular}{|c|c|c|c|}
\hline Factor 1 & Factor 2 & \\
\hline & Cluster 2.1 & $\ldots$ & Cluster 2.r \\
\hline Cluster 1.1 & $\mathrm{D}_{1,1} \subseteq \mathrm{D}_{\mathrm{i}}$ & $\ldots$ & $\mathrm{D}_{1, \mathrm{r}} \subseteq \mathrm{D}_{\mathrm{i}}$ \\
\hline$\ldots$ & $\ldots$ & $\ldots$ & $\ldots$ \\
\hline Cluster 1.v & $\mathrm{D}_{\mathrm{v}, 1} \subseteq \mathrm{D}_{\mathrm{i}}$ & $\ldots$ & $\mathrm{D}_{\mathrm{v}, \mathrm{r}} \subseteq \mathrm{D}_{\mathrm{i}}$ \\
\hline
\end{tabular}

Table 1: Dataset allocation table

$D_{1,} D_{1} \cup \ldots \cup D_{1, r} \cup \ldots \cup D_{v, 1} \cup \ldots \cup D_{v, r}=D_{i}$,
$\forall v, r \in N$

The essence of the dataset allocation table is that it "divides" each productivity dataset in specific operational scenarios, i.e. pairwise combinations of operational factors. Hence, each table cell represents a unique operational setting within the designated clusters, thus highlighting the contextual meaning of each data point. There is no standard rule as to how many data points there should be in each cluster. It is evident, however, that as the sample size increases, the inferences derived from each cluster will be more valid. For indicative reasons, the table presented before has two dimensions. However, the analysis could be easily extended to incorporate three or more parameters, where each cluster would be illustrated in a tree-structure.

The next step is the definition of the "Baseline Reference Conditions" $(B R C)$, namely the operational conditions under which every operational coefficient can be neglected, as it is supposed that it does not affect productivity. On a theoretical basis, this means that when certain conditions are met, then $\mathrm{p}_{\mathrm{i}}=1.00, \forall_{\mathrm{i}} \in \mathrm{N}$ (see Equation 2), and, consequently, theoretical and effective productivity coincide (Panas and Pantouvakis 2010). In essence, the baseline reference conditions represent a specific operational scenario, or, if seen in relation to Table 1 , the $B R C$ are associated with a certain table cell. The choice of the BRC scenario depends on the analyst's preference. A practical rule though would be for the BRC scenario to be specified as the table cell with most data points, since it will then represent the operational scenario most frequently met on site (see Equation 6).

Upon the establishment of the BRC, the "Baseline Reference Metrics" (BRM) are defined, namely the productivity values which correspond to the baseline reference scenario. In the absence of actual data, BRM can be extracted from estimation handbooks or from a company's historical record. When field measurement data is available, then the BRM is directly associated with the dataset allocation table, since it is equal to the average of the BRC cell's values (see Equation 7).

$B R C=\max \left\{D_{1,1}, \ldots, D_{1, r}, \ldots, D_{v, 1}, \ldots, D_{v, r}\right\}$

$B R M=\left\langle\max \left\{D_{1,1}, \ldots, D_{1, r}, \ldots, D_{v, 1}, \ldots, D_{v, r}\right\}\right\rangle$

For example, if it is assumed that in Table 1 the majority of the data points are found in w-th row and z-th column, then the cell containing the $D_{w, z}$ dataset is considered as representing the baseline reference conditions, as shown in Table 2, below.

In this manner, the BRM is estimated as the average value of all data points contained in the $D_{w, z}$ dataset (see Equation 8):

$B R M=\left\langle D_{w, z}\right\rangle$

The analysis concludes with the calculation of the efficiency multipliers, as dictated by Equation 2. The coefficients are calculated for every cluster in a v-by-r matrix as shown below (see Equation 9): 


\begin{tabular}{|c|c|c|c|c|c|}
\hline Factor1 & Factor 2 & & & & \\
\hline & Cluster 2.1 & $\ldots$ & Cluster $2 . z$ & $\ldots$ & Cluster 2.r \\
\hline Cluster 1.1 & $\mathrm{D}_{1,1} \subseteq \mathrm{D}_{\mathrm{i}}$ & $\ldots$ & $\mathrm{D}_{1,2} \subseteq \mathrm{D}_{\mathrm{i}}$ & $\ldots$ & $\mathrm{D}_{1, \mathrm{r}} \subseteq \mathrm{D}_{\mathrm{i}}$ \\
\hline$\ldots$ & $\ldots$ & $\ldots$ & $\ldots$ & $\ldots$ & $\ldots$ \\
\hline Cluster 1.w & $\mathrm{D}_{\mathrm{w}, 1} \subseteq \mathrm{D}_{\mathrm{i}}$ & $\ldots$ & $\mathrm{D}_{\mathrm{w}, \mathrm{z}} \subseteq \mathrm{D}_{\mathrm{i}}$ & $\ldots$ & $\mathrm{D}_{\mathrm{w}, \mathrm{r}} \subseteq \mathrm{D}_{\mathrm{i}}$ \\
\hline$\ldots$ & ... & $\cdots$ & $\cdots$ & $\cdots$ & ... \\
\hline Cluster 1.v & $\mathrm{D}_{\mathrm{v}, 1} \subseteq \mathrm{D}_{\mathrm{i}}$ & $\ldots$ & $\mathrm{D}_{\mathrm{v}, \mathrm{z}} \subseteq \mathrm{D}_{\mathrm{i}}$ & $\ldots$ & $\mathrm{D}_{\mathrm{v}, \mathrm{r}} \subseteq \mathrm{D}_{\mathrm{i}}$ \\
\hline
\end{tabular}

Table 2: Baseline Reference Metrics (BRM) specification table

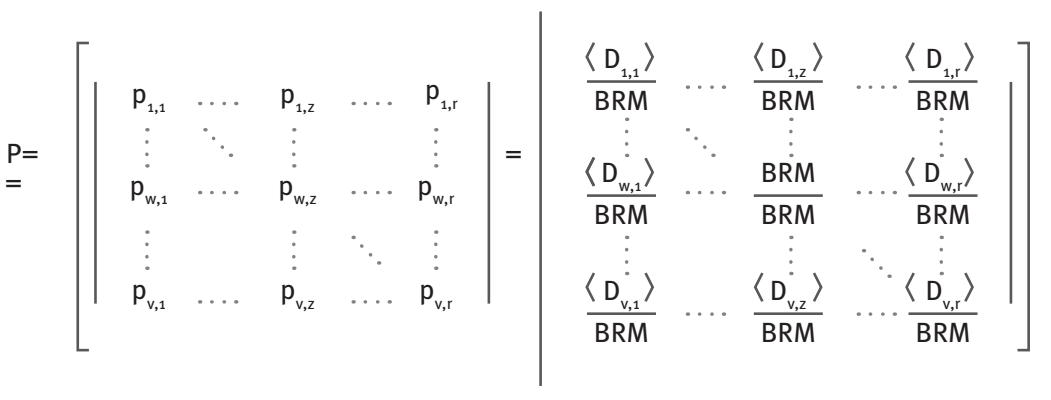

It is evident that the BRM efficiency multipliers are always equal to 1.00 . In addition, the efficiency multipliers of the BRM row $\left(\mathrm{p}_{\mathrm{w}, 1}, \ldots, \mathrm{p}_{\mathrm{w}, \mathrm{z}}, \ldots \mathrm{p}_{\mathrm{w}, \mathrm{r}}\right)$ and column $\left(p_{1, z}, \ldots, p_{w, z}, \ldots p_{v, z}\right)$ vectors indicate the variation in productivity under the separate influence of either factor 2 or factor 1 respectively. This is particularly important for the establishment of a valid experimental framework, in case the analysis should be conducted under the prism of a sole operational factor. In other words, if the effect of operational factor 1 on productivity were to be examined independently of the influence of any other operational factor, then the analyst should conduct field measurements for different clusters of factor 1 , provided that the values of factor 2 would be strictly confined within cluster 2.z. The rest of the matrix elements indicate the variation in productivity under the combined effect of both factors.

When all $p_{i}$ coefficients have been estimated, comparative analyses can be conducted to evaluate the intra-row or intra-column variation of the BRM,
New multipliers estimation: Case Study of concrete pavement construction

A practical implementation of the developed concepts is presented in the following sections, so as to demonstrate the applicability of the analysis methodology. Data were collected through work studies of actual paving operations for the construction of a container terminal infrastructure over eight months, taking place in two different periods (2011 and 2013). Direct observation and video recording were used as primary data elicitation instruments. Secondary data were gathered by open interviews with senior project management staff, construction managers and site personnel, as well as by studying project documentation (drawings, quantity take-offs, progress payment orders, labour hours logs). All data points have been grouped in specific datasets, while the scope of measurements for each variable is denoted by the minimum and maximum values described before. In total, 46 data points have been collected representing on-site workday measurements of concrete paving productivity, expressed in work-hours per cubic meter of placed concrete $\left(\mathrm{wh} / \mathrm{m}^{3}\right)$.

\section{Phase 1: Activity definition and data} elicitation

Although measurements have been collected for all subtasks of the concrete pavement process (see Concrete paving operations), the analysis will be focused on the concrete layering subtask $(n=1)$, for brevity reasons. The 
productivity factors which were initially screened as candidate operational factors were the working width and length, the concrete layer thickness, the concrete type (fiber-reinforced or plain) and the gang size. Based on the visualization of the pairwise correlations between these response variables through a scatterplot matrix and the implementation of the backward stepwise selection technique, it was decided that the concrete paving operations should be better examined by taking into account the following operational factors: working width and length. It is evident that each one of the aforementioned factors holds a certain set of attributes. The working width (w) holds a minimum value of $4 \mathrm{~m}$ which represents the min working range of the laser screed and maximum of $33 \mathrm{~m}$. The working length $(\mathrm{l})$ ranges between $15 \mathrm{~m}-210 \mathrm{~m}$ and represents the lane length that is worked by a crew on a given workday. On the basis of the operational factors described before, the collected data will be divided according to their attributes in specific clusters, so as to enable their computational processing, as will be shown in the next sections.

\section{Phase 2: Productivity model \\ generation}

This section presents the results of the analysis for the productivity model generation process, through the implementation of the statistical regression technique. The working width and length are the explanatory variables, whereas productivity is the dependent variable. The first step would be the scanning of the data for outlying values and their examination to see if they are valid observations. An outlier analysis was conducted by the use of three statistical metrics (Mahalanobis distance, jackknife distances, T2 statistic) and five data points were excluded from the model. After the outliers' identification and since all data lie within the designated margins of Table 1 , the next step is the multiple regression model generation, with working width and length being the predictor variables and layering productivity considered as the response variable. The provision of the source data is omitted due to space limitation, however the results of the model fitting process showed that the model has an R-square value 0.68432 , which represents the coefficient of multiple determination that measures the proportional reduction of total variation in producitivity using working width and length as independent variables. In other words, it represents the total variability in productivity explained by working width and length. R-squares values of >0.60 imply that the data correlation is positive and strong and, thus, acceptable (Kutner et al., 2005). The analysis of variance yielded an observed significance probability (Prob>F) of $<0.0001$ for width and length, which is significant at the 0.05 level. The Prob>|t| metric is $<0.0001$ for the working width and length, as well as for the intercept, which means that $b_{1} \neq 0, b_{2} \neq 0$ and $b_{0} \neq 0$ at $99 \%$ confidence respectively. Ultimately, the following productivity model is used (see Equation 11):

$\mathrm{Q}_{\text {eff }}=0.4900881-0.007678 \times[$ Width] $-0.001332 \times$ [Length], [wh/m³]

Figure 3 illustrates the plotted regression model as a function of working length, for different width values, whereas the respective validation chart is depicted in Figure 4. The mean absolute percentage error is $10.98 \%$, which shows that the model values fit actual productivity data adequately.

It should be noted that, in principle, the determination of a quantitative relationship for estimating construction productivity with sufficient statistical confidence requires the inclusion of a large database of field measurements (AbouRizk et al., 2001; Thomas and Yiakoumis, 1987). As such, the developed model is at a preliminary stage, since its derivation has been based on a limited number of collected datapoints. However, its face validity has been successfully tested in a real construction setting and it is currently extended with the inclusion of more data, in order to increase its predictability and stability. Besides, the model's main purpose is to serve as an instrument to exemplify the applicability of the proposed methodology.

Phase 3: Estimation and comparative evaluation of efficiency multipliers

\section{Specification of aggregate}

efficiency multipliers for all involved operational factors

After the validation of the developed model, the "efficiency multipliers determination" phase initiates as depicted in Figure 1. The two basic operational factors are working width and length and their data clusters are specified in such a way, so that the all data points within a single dataset are distributed accordingly. The dataset allocation table is formed as follows (see Table 3).

Following the empirical rule expressed by Equation 6, the BRC scenario is represented by the $D_{3,1}$ cell. In this case, the $B R C$ scenario is defined as the one corresponding to a working width of $21 \mathrm{~m}$ $35 \mathrm{~m}$ and working length of om-5om. Consequently, the BRM for concrete layering is established as the average productivity value of the data points contained in that cell, thus yielding $B R M=0.22 \mathrm{wh} / \mathrm{m}^{3}$. The matrix for the calculation of the $p_{i}$ coefficients and the subsequent estimation of the effective productivity values is formulated as follows (see Equations 12 and 13):

Since two operational factors are taken into account, then productivity can be examined either under the combined influence of the working width and length, which is represented by the aggregate efficiency multipliers of the matrix in Equation 12, or separately for each factor. For the latter case, the isolated effect of the working width 
$P=\left|\begin{array}{ccc}2.06 & 1.39 & 0.39 \\ 1.49 & 1.26 & 1.07 \\ 1.00 & 0.88 & 0.74\end{array}\right|$

$Q_{\text {eff }}=Q_{\text {eff }} \times P=0.22 \times P=0.22 \times\left|\begin{array}{ccc}2.06 & 1.39 & 0.39 \\ 1.49 & 1.26 & 1.07 \\ 1.00 & 0.88 & 0.74\end{array}\right|=\left|\begin{array}{lll}0.45 & 0.31 & 0.20 \\ 0.33 & 0.28 & 0.24 \\ 0.22 & 0.19 & 0.16\end{array}\right|$

\subsubsection{Specification of seperate efficiency multipliers for}

specific operational factors

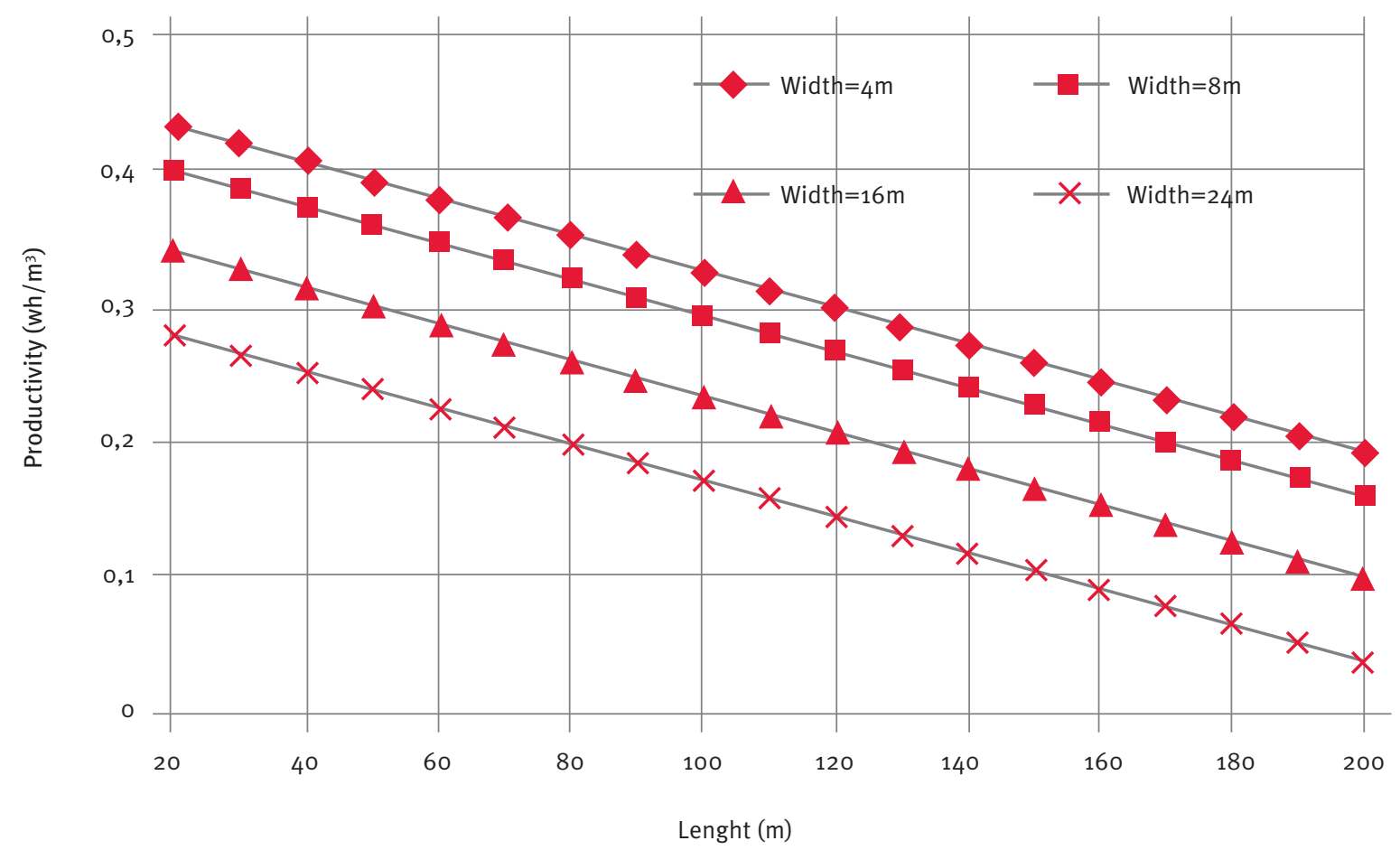

Figure 3: Concrete layering productivity estimation chart.

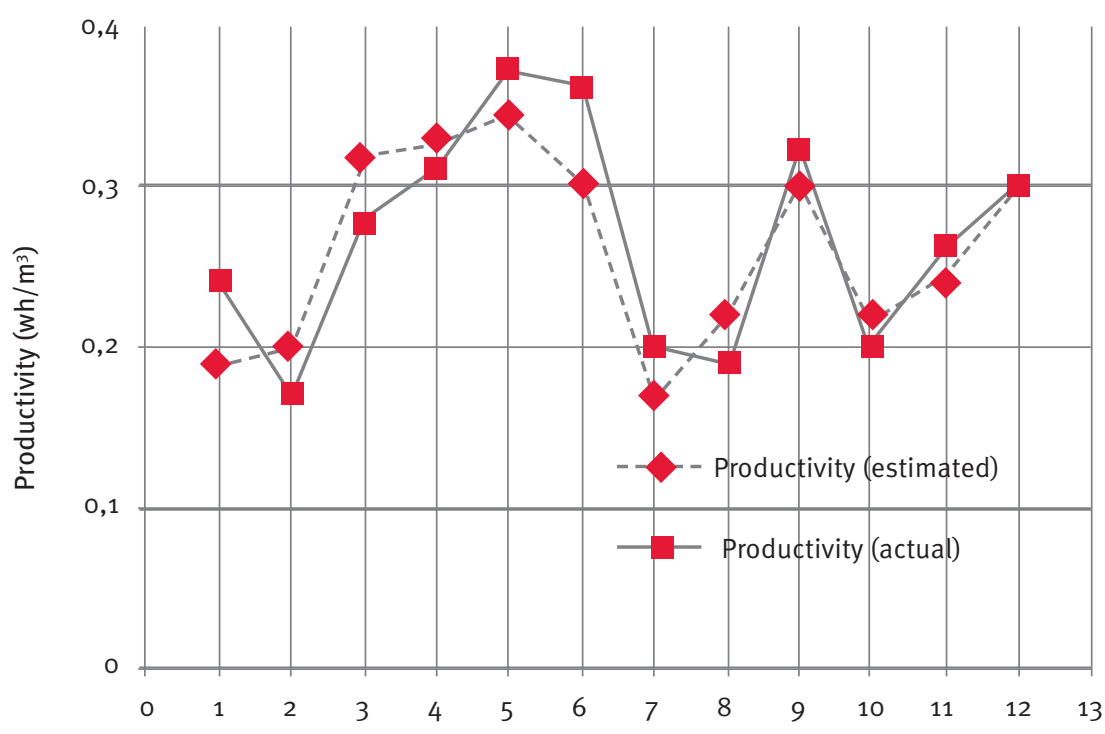

Figure 4 . Concrete layering productivity validation chart. 


\begin{tabular}{|c|c|c|c|}
\hline Width [m] & Length [m] & & $101-215$ \\
\hline & $0-50$ & $51-100$ & $3,8,9,10,11,12,13$ \\
\hline $0-10$ & 16,32 & 1,15 & 19 \\
\hline $11-20$ & $20,21,23,24,25,29,30,35$ & $4,7,14,34,37,42,46$ \\
\hline $21-5$ & $22,26,27,28,31,33,38,39,40,41,43,45$ & 36 & 18 \\
\hline
\end{tabular}

Table 3: Baseline Reference Metrics (BRM) specification table

on productivity is expressed by all $p_{i}$ coefficients in the first column of the matrix, whereas, in a similar fashion, the effect of the working length is denoted by the equivalent values of the third row of the matrix. Let us assume that the objective is the study of the impact that each factor (i.e. width and length) has on productivity. Then, Equation 2 would be modified into Equation 14 as follows:

$$
Q_{\text {eff }}=Q_{\text {th }} \times p_{\text {length }} \times p_{\text {width }} \text { (14) }
$$

where: $p_{\text {length/width }}=$ the dedicated efficiency multiplier representing the quantitative impact of the working length and width for the adjustment of theoretical to effective productivity.

In other words, $p_{\text {length }}$ illustrates productivity's sensitivity solely to the available length under no other influence of any other operational factor. Since the aggregate multiplier of Equation 12 for width values within the $21 \mathrm{~m}-35 \mathrm{~m}$ range and respective length values within the om-50m range is 1.00 , this means that $\mathrm{p}_{\text {length }} \times \mathrm{p}_{\text {width }}=$ $1.00 \times 1.00=1.00$. As such, both factors are assumed at a baseline reference condition for that particular operational scenario. The statistically valid examination of the length's effect on productivity would require $p_{\text {width }}$ to be held constantly equal to 1.00 , so that $Q_{\text {eff }}=Q_{\text {th }} \times p_{\text {length }} \times 1.00=Q_{\text {th }} \times p_{\text {length }}$. This means that $p_{\text {length }}$ should be examined along the third row of the multipliers matrix (see Equation 12). Hence, for a constant working width in the region of $21 \mathrm{~m}-35 \mathrm{~m}$, the effective productivity will incrementally improve by approx. $13.64 \%$ and $15.79 \%$ respectively, as the working length increases from om-5om
$\left(Q_{\text {eff }}=0.22 \times 1.00=0.22 \mathrm{Wh} / \mathrm{m}^{3}\right)$, to $51 \mathrm{~m}$ $100 \mathrm{~m}\left(\mathrm{Q}_{\text {eff }}=0.22 \times 0.88=0.19 \mathrm{wh} / \mathrm{m}^{3}\right)$ and ultimately to $101 \mathrm{~m}-215 \mathrm{~m}\left(\mathrm{Q}_{\text {eff }}=0.22\right.$ $\left.\times 0.74=0.16 \mathrm{wh} / \mathrm{m}^{3}\right)$, as shown by the last row of the respective productivity estimation matrix (see Equation 13). In the same fashion, the equivalent efficiency multiplier for width ( $p_{\text {width }}$ ) would be estimated for a working length less than $50 \mathrm{~m}$, so that $\mathrm{Q}_{\text {eff }}=$ $\mathrm{Q}_{\text {th }} \times 1.00 \times \mathrm{p}_{\text {width }}=\mathrm{Q}_{\text {th }} \times \mathrm{p}_{\text {width }}$. In that sense, it is found that productivity is decreased by $\sim 50.00 \%$ for a working width in the region of $11 \mathrm{~m}-20 \mathrm{~m}$ $\left(Q_{\text {eff }}=0.22 \times 1.49=0.33 \mathrm{wh} / \mathrm{m}^{3}\right)$ and, subsequently, further diminishes by $36.36 \%$ for widths lower than $10 \mathrm{~m}$ $\left(Q_{\text {eff }}=0.22 \times 2.06=0.45 \mathrm{wh} / \mathrm{m}^{3}\right)$.

In any other case, the comparative evaluation of the efficiency multipliers demands for their gradual variation to be explicitly taken into account, since the analysis is shifting away from the BRC state into other cells, which denote different operational scenarios. Thus, if the length is supposed to be within $51 \mathrm{~m}$ $100 \mathrm{~m}$, then the effective productivity is first adjusted by the efficiency multiplier $\mathrm{p}_{\text {length }}$, in order to shift from the BRC state to the $51 \mathrm{~m}-100 \mathrm{~m}$ category: $Q_{\text {eff }}=Q_{\text {th }} \times p_{\text {length }}=0.22 \times 0.88=0.19 \mathrm{Wh} /$ m3. The estimation of effective productivity values within that length range requires the calculation of the partial width multipliers as follows: For a width ranging from $11 \mathrm{~m}-20 \mathrm{~m}, \mathrm{p}_{\text {width }}=1.26 /$ $0.88=1.43$ and, hence, $Q_{\text {eff }}=Q_{\text {th }} \times p_{\text {length }}$ $\times \mathrm{p}_{\text {width }}=0.22 \times 0.88 \times 1.43=0.28 \mathrm{wh} /$ m3. Finally, for widths lower than $10 \mathrm{~m}, \mathrm{p}_{\text {width }}=1.39 / \mathrm{o} .88=1.58$, so $\mathrm{Q}_{\text {eff }}$ $=0.22 \times 0.88 \times 1.58=0.31 \mathrm{wh} / \mathrm{m} 3$. Note, that the last result could be reached directly, if the aggregate efficiency multiplier was used for width values within om-10m and length values within $51 \mathrm{~m}$ 100m (see Equation 12):

$\mathrm{Q}_{\text {eff }}=\mathrm{Q}_{\mathrm{th}} \times \mathrm{p} 1,2=0.22 \times 1.39=$ $0.31 \mathrm{wh} / \mathrm{m} 3$. If adequate data is available, then the effect of $p_{\text {length }}$ and $p_{\text {width }}$ can be generalised and aid estimators in adjusting their estimates under different operational scenarios. It should be noted, though, that the computed efficiency multipliers have been derived from a rather small sample. It is logical, that as more data points are being added to the sample, the predictive capability of the efficiency multipliers is going to be improved. However, the validity of the estimation process per se is independent of the sample size and should only abide with the statistical inferences of the experimental framework, as described in the research methodology.

\section{Known multipliers}

\section{corroboration: The case of the} hydraulic excavator

In view of the analysis presented in the previous section, it would be useful to examine how known efficiency estimators can be corroborated within a specific operational setting. Equation 3 as defined by BML (1983) for the estimation of the hourly productivity of the hydraulic excavator will be used in this section. More specifically, let us assume that the method statement for an excavation operation dictates that the hydraulic excavator should operate at a swing angle of $45^{\circ}$ and at the depth of $5 \mathrm{~m}$. First, the baseline reference conditions are represented 
by a swing angle of $90^{\circ}\left(\mathrm{p}_{\text {swing }}=1.00\right)$ and an excavation depth of $1.00 \mathrm{~m}$ $\left(p_{\text {depth }}=1.00\right)$. In that view, the actual on-site measurement of the $p_{\text {swing }}$ efficiency multiplier should be conducted for excavation depths in the area of $1.00 \mathrm{~m}$, whereas the respective determination of the $p_{\text {depth }}$ efficiency multiplier should stem from measurements at swing angles equal to $90^{\circ}$. Hence, taking into account the exact operating conditions, the sensitivity analysis for the variation of the $p_{\text {swing }}$ cannot be undertaken at the baseline reference conditions. In that case, first the $p_{\text {depth }}$ is estimated as $p_{\text {depth }}=0.0043$ $\times 52-0.0622 \times 5+1.0618=0.86$ and, subsequently, productivity variation is examined according to the mathematical formula $Q_{\text {eff }}=Q_{\text {th }} \times p_{\text {swing }} \times 0.86$, for different values of $p_{\text {swing }}$. In a similar fashion, the examination of the sensitivity for the $p_{\text {depth }}$ multiplier demands the estimation of the $p_{\text {swing }}$ for $45^{\circ}$ as $p_{\text {swing }}=4 \times 10-6 \times 452-0.0024 \times 45+$ $1.1824=1.08$. Then, the productivity estimation formula is $Q_{\text {eff }}=Q_{\text {th }} \times p_{\text {depth }}$ $\times 1.08$, for different values of $p_{\text {depth }}$. In that sense, the values provided in the estimation handbooks can be critically evaluated and their applicability can be examined according to their ability to reflect the actual on-site conditions.

\section{Discussion}

This section presents the main inferences emerging from the study. This study research contribution is assessed along three pillars:

1. Methodological framework: The study has demonstrated that the establishment of a structured approach for assessing construction productivity may be translated into specific and practical steps that provide full control over the estimating process. In addition, the importance of the contextual framework has been highlighted, since the examination of different operational scenarios suggests the implementation of strict procedures in handling the involved efficiency multipliers, in order for the analysis to yield valid and comparable results. The study might be criticized for its limited data sample, since, indicatively, the efficiency multiplier in the second row and third column of the matrix in Equation 12 has been derived from a single data point, which is, obviously, not representative of the activity under study. Consequently, for the cells that contain little data, two possible remedial actions would be either to merge clusters, so as to end up with more data points in all cells, or use the validated regression model in order to "generate" data points within the selected clusters.

2. Estimation of new efficiency multipliers: The analytical determination of the efficiency multipliers enables the dynamic parameterization of the estimation process, since the analysis can be easily shifted from the "baseline reference scenario" to any other operational settings. Thus, the change in the working conditions is explicitly quantified from a productivity standpoint, because each efficiency multiplier is directly associated with a specific operational factor: in our case, these were the working width and length for concrete layering in paving operations and the swing angle and digging depth for the excavation operations. On any case, it must be highlighted that the derived empirical models should be constrained to their factors and respective attributes, as defined in the dataset allocation table (e.g. see Table 3 for concrete layering operations) and not be generalised beyond that scope.

3. Comparative evaluation of efficiency multipliers: The research contribution stemming from the performance ratio (PR) decomposition was twofold: first, it enabled the isolation of the selected factors' impact on productivity. The applied approach can be easily extended to include more than two factors, depending on the estimator's judgment. Secondly, this is the first study to evaluate the effect of the contextual framework on a productivity sensitivity analysis against a specific factor. The term "contextual framework" denotes the state assumed by the rest of the productivity factors not included in the sensitivity analysis. Therefore, it is evident that it would not suffice to vary a specific productivity factor's values and estimate its effect on productivity, if the estimator has no knowledge or control over the varying state of the other productivity factors. It must be ensured that all other parameters are at a "baseline reference" state, so as for their impact to be neglected. This means that pi would be equal to 1.00 for all productivity factors, except the one currently investigated. In any other case, the shift from the "baseline reference" state to other operational scenarios must be carefully conducted, as was shown by the case study results.

An additional point is that the proposed framework may be implemented for both labor- and equipment-intensive operations, as long as productivity estimation can be formalized mathematically in an explicit, quantifiable way. The qualitative examination of productivity influencing factors requires a different methodological approach, which, however, falls out of the scope of this study.

\section{Conclusions}

This study presented an investigation into the estimation and comparative evaluation of new efficiency multipliers and the corroboration of known efficiency multipliers for a given construction activity. The research did not pursue the development of just another productivity model. The main research 
contribution is the development of a structured estimation approach, whose functional characteristics allow the quantitative assessment of the impact that dynamically changing operational factors have on construction productivity. In addition, this research adds to the existing body of knowledge since it (i) formulates the computational framework that allows the specification of statistically valid aggregate efficiency multipliers that quantify the performance ratio (PR) impact on productivity and (ii) implements the concept of the "baseline reference conditions", so as to estimate separate efficiency multipliers for each involved factor. The case study results indicate that productivity is affected more by width rather than length variations. In addition, a larger working area increases productivity for a given working width or length. It is believed that the proposed approach supports estimators in corroborating or improving the results of past productivity studies and efficiency multipliers derived from historical data. Future research is suggested towards the investigation of other activities, in order to further validate the proposed approach, as well as automate the proposed methodological framework, through the development of a computer-based estimation tool.

\section{References}

AbouRizk, S., Knowles, P. and Hermann, U. R. (2001). "Estimating labor production rates for industrial construction activities", Journal of Construction Engineering and Management, Vol. 127, No. 6, pp. 502-511.

BML (1983), Handbuch BML: Daten für die Berechnung von Baumaschinen-Leistungen, Zeittechnik Verlag, Neu-Isenburg.

Caterpillar (2014), Caterpillar Performance Handbook, 44th edition, Caterpillar Inc., Illinois, USA.

Hasan, S., Bouferguene, A., Al-Hussein, M., Gillis, P. and Telyas, A. (2013), "Productivity and $\mathrm{CO}_{2}$ emission analysis for tower crane utilization on high-rise building projects", Automation in Construction, Vol. 31, pp. 255-264.

Jang, H., Kim, K., Kim, J. and Kim, J. (2011), "Labour productivity model for reinforced concrete construction projects", Construction Innovation, Vol. 11, No. 1, pp. 92-113.

Kiziltas, S. and Akinci, B. (2009), “Contextual information requirements of cost estimators from past construction projects", Journal of Construction Engineering and Management, Vol. 135, No. 9, pp. 841-852.

Komatsu (2009), Specifications and Application Handbook, 3oth edition, Komatsu, Japan.

Kutner, M.H., Nachtsheim, C.J., Neter, J. and Li, W. (2005), Applied linear statistical models, Mc-Graw Hill, New York.

Lambropoulos, S., Manolopoulos, N. and Pantouvakis, J.P. (1996), “SEMANTIC: Smart EarthMoving ANalaysis and estimation of Cost”, Construction Management and Economics, Vol. 14, No. 2, pp. 79-92.

Moselhi, O. and Khan, Z. (2010), "Analysis of labour productivity of formwork operations in building construction", Construction Innovation, Vol. 10, No. 3, pp. 286-303.

Moselhi, O. and Khan, Z. (2012), "Significance ranking of parameters impacting construction labour productivity", Construction Innovation, Vol. 12, No. 3, pp. 272-296.

O’Connor, J.T. and Huh, Y. (2006), “Crew production rates for contract time estimation: Beam erection, deck, and rail of highway bridges", Journal of Construction
Engineering and Management, Vol. 132, No. 4, pp. 408-415.

Panas, A. and Pantouvakis, J.P. (2010), “Comparative analysis of operational coefficients' impact on excavation operations", Engineering Construction and Architectural Management, Vol. 17, No. 5, pp. 461-475.

Panas, A. and Pantouvakis, J.P. (2011), “Multiattribute regression analysis for concrete pavement productivity estimation", Organization, Technology and Management in Construction: An International Journal, Vol. 3, No. 2, pp. 289-295.

Panas, A. and Pantouvakis, J.P. (2014), "Simulation-Based and Statistical Analysis of the Learning Effect in Floating Caisson Construction Operations", Journal of Construction Engineering and Management, Vol. 140, No. 1, 04013033.

Pantouvakis, J.P. and Panas, A. (2013), "Computer simulation and analysis framework for floating caisson construction operations", Automation in Construction, Vol. 36, pp. 196-207.

Song, L. and AbouRizk, S.M. (2008), “Measuring and modeling labor productivity using historical data." Journal of Construction Engineering and Management, Vol. 134, No. 10, pp. 786-794.

Thomas, H.R. and Yiakoumis, I. (1987), "Factor model of construction productivity." Journal of Construction Engineering and Management, Vol. 113, No. 4, pp. 623-639. 